\title{
Otimização do Custo de Transporte na Distribuição-Armazenagem de Açúcar
}

\author{
Emerson Carlos Colin \\ Escola Politécnica da USP - Departamento de Engenharia de Produção \\ Rua Catolândia, 765, CEP 02839-000, São Paulo - SP \\ e-mail:emerson@usp.br \\ Flávio A. M. Cipparrone \\ Escola Politécnica da USP - Departamento de Engenharia Eletrônica \\ Av. Prof. Luciano Gualberto, trav. 3, 158, CEP 05508-900, São Paulo-SP \\ e-mail: cippafla@lps.usp.br \\ Tamio Shimizu \\ Escola Politécnica da USP - Departamento de Engenharia de Produção \\ Av. Prof. Almeida Prado, trav. 2, 128, 20 andar, CEP 05508-900, Säo Paulo - SP
}

\section{Resumo}

Este trabalho apresenta um caso de aplicação da programaçāo linear em um sistema logístico de distribuiçāo de açúcar. Para um intervalo de 13 meses, o sistema leva em consideração as capacidades de estocagem dos diversos depósitos, a produçāo da usina, assim como os custos envolvidos no transporte do açúcar. Apresentam-se detalhes da modelagem realizada bem como os resultados obtidos. O trabalho é finalizado com sugestōes para o desenvolvimento de possíveis extensōes nesse tipo de ambiente.

\section{Abstract}

This work presents a specific model of linear programming application in a logistic distribution system. This system takes into account the storage capacity of the several warehouses, usine production, and transportation costs involved in sugar transportation in a period of 13 months. We present details of the modeling process and how the results were obtained. As a final discussion, the work suggests some extensions of the problem for this kind of environment.

Palavras Chaves:

Distribuição Logística, Otimização, Aplicação de Programação Linear.

Keywords:

Logistic Distribution, Optimization, Linear Programming Application.

\section{Introdução}

A utilização de modelagem matemática em sistemas de produção brasileiros tem se tornado freqüente. Podemos citar exemplos recentes como Taube-Netto (1996) e Yoshizaki et al. (1996). Uma das possíveis razões para que isso aconteça é que a classe empresarial esteja interessada na eficácia operacional, ou melhor dizendo, esteja sendo forçada a se interessar devido aos efeitos da competição mais acirrada e mais profissional. Outro fator quer pode ser ressaltado é que devido à pequena utilização de ferramentas desse tipo no Brasil, surjam oportunidades de melhoria simples e que não necessitem de desenvolvimento muito além do disponível na literatura clássica, para sua 
solução e implementação.

O presente trabalho é mais um exemplo nesse sentido - guardadas as devidas proporções , onde a utilização de uma modelagem simples e já bastante conhecida, poderia proporcionar uma economia de aproximadamente US\$75.000,00/ ano, representando $1 \%$ dos custos totais de transporte da empresa em consideração. A princípio o modelo não foi implementado efetivamente, mas sim em paralelo ao sistema de tomada de decisões convencional, visando dessa forma ser comparado e avaliado com relação aos resultados que poderiam ser alcançados. Esse tipo de abordagem não é incomum na literatura, conforme pode ser observado em Fisher; Raman (1996).

Na seção 2 descrevemos o sistema logístico de uma forma geral. A seção 3 apresenta a modelagem matemática do sistema. Na seção 4 mostramos alguns detalhes da implementação computacional, enquanto que na seção 5 apresentamos a avaliação econômica da utilização do modelo. Finalizamos o trabalho com discussões interessantes para empresas que desejem fazer implementações semelhantes e mais completas.

\section{O Problema Analisado}

O sistema de distribuição em análise leva em consideração a produção da usina de açúcar que é dependente da época de colheita, de um depósito geral que fica situado próximo à usina produtora e de oito depósitos de distribuição que ficam próximos aos mercados consumidores. Um esquema gráfico do mesmo é apresentado na Figura 1. O planejamento da produção é iniciado com a definição da quantidade de cana-de-açúcar a ser plantada pelo menos dois anos antes da colheita. Podemos perceber com isso que a produção de uma determinada usina produtora só pode ser alterada a longo prazo. Além disso, por uma questão de sazonalidade da colheita da cana, a produção acontece apenas durante sete meses em cada ano, geralmente de maio até novembro. Deve-se notar que como as vendas são feitas o ano todo, e como a produção é de apenas sete meses, é de fundamental importância que haja um sistema eficiente que possa administrar esse problema de distribuição e armazenagem. A inexistência de um estoque para atendimento na entressafra pode levar a uma perda de clientes para a concorrência.

A colheita de cana acontece obviamente nos meses onde a plantação permite um maior rendimento de açúcar por quantidade de cana plantada, ou seja, nos meses em que ocorre uma maior maturação. Em realidade, poderíamos dizer que há um ponto ideal de colheita no que tange a essa maturação (no mês de setembro de acordo com Tomiya, 1994, p. 11), mas não é possível aplicar uma quantidade de recursos que explore esse ponto ideal. Existem restrições com relação ao recrutamento da mão-de-obra utilizada na colheita e com relação à própria capacidade dos equipamentos utilizados na produção.

Outro fato interessante é que a produção é dependente de fatores climáticos como a chuva e o sol. No período de entressafra, a chuva é importante para o crescimento da cana, enquanto que no período de safra, o excesso de chuva pode levar a uma cana com água em excesso e por conseqüência pequena concentração de açúcar por unidade de massa colhida - ou seja, grande parte da colheita é água -. Além disso, como a colheita é altamente baseada em operações manuais, a chuva impõe dificuldades na própria operação de colheita e carregamento de caminhões. Parece-nos que há um grande espaço para a utilização de sistemas mais automatizados ou até mesmo mais mecanizados que possam garantir uma produção mais contínua e robusta. 


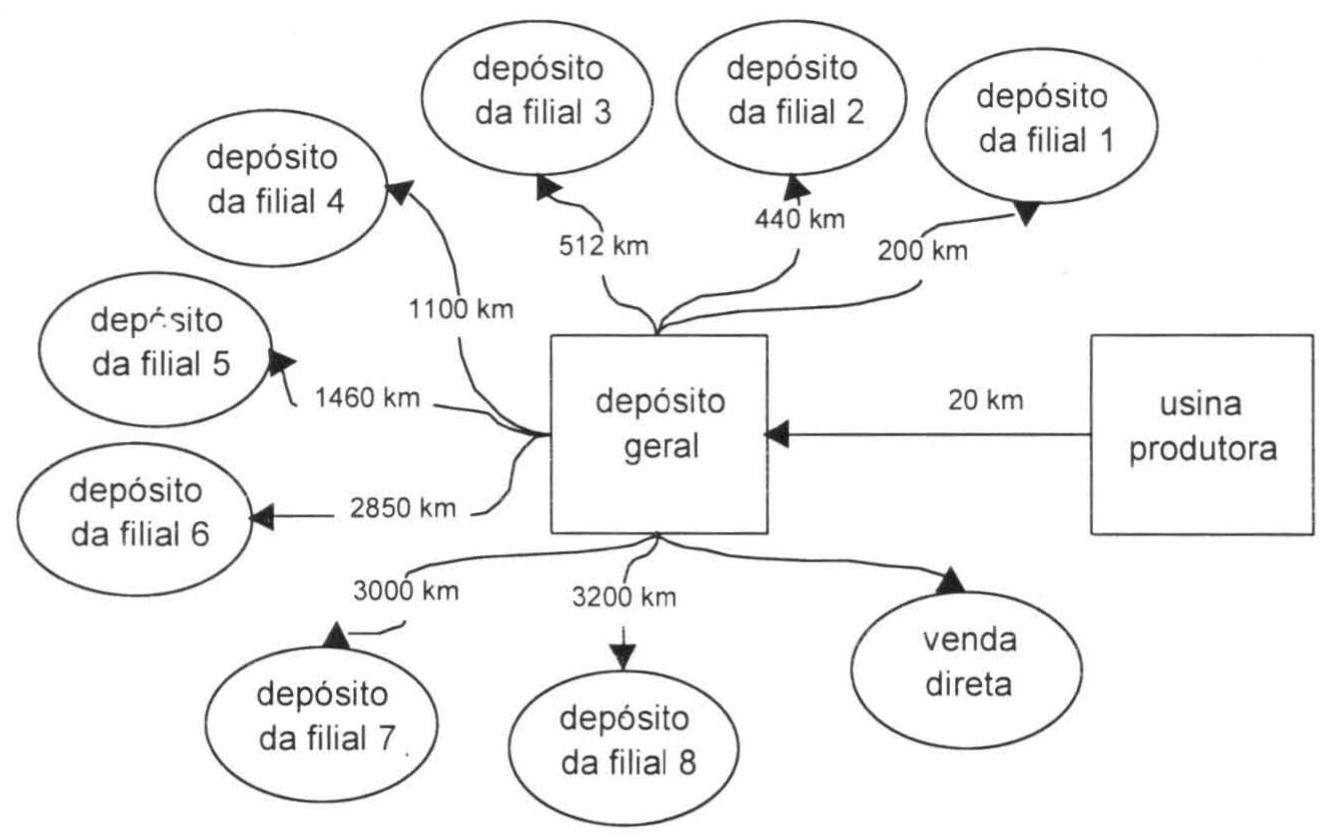

Figura 1: sistema de prodıção-armazenagem-distribuição de açúcar

Após a produção, o açúcar é enviado ao depósito geral que fica a $20 \mathrm{~km}$ da usina produtora. Como esse envio é necessário, pode-se dizer que faz parte do processo de produção e conseqüentemente os custos nesse transporte não foram considerados no estudo.

Estando o açúcar no depósito geral, existem 3 decisões que podem ser tomadas em um determinado instante: (a) manter o açúcar no estoque até um período futuro, (b) vender o açúcar diretamente no mercado loual ou, (c) enviar o produto para o depósito de uma das filiais. Para os depósitos das filiais ficam apenas duas decísões: (a) vender o açúcar no período atual e, (b) vender o açúcar em um período futuro.

Em nosso modelo, as decisões de interesse serão aquelas relacionadas com o transporte. Como todo o transporte é terceirizado, seus custos são sazonais devido à concorrência em certos períodos com safras de outras culturas e mesmo com as de açúcar de outras usinas.
Dado esse ambiente, desejamos saber qual a quantidade, e em que data enviar o açúcar para as filiais, de forma que o custo de transporte seja minimizado e que as demandas das filiais e a demanda direta sejam atendidas. Além disso outras restrições como as de capacidade de armazenagem e de produção da usina devem ser consideradas.

\section{A Modelagem Utilizada}

Para a modelagem do sistema, utilizamos uma formulação clássica da programação linear para problemas de transporte. O problema pode ser considerado como um problema de alocação. A notação utilizada é a que segue abaixo:

Índices:

$\mathrm{i}=$ índice dos depósitos;

$\mathrm{j}=$ indice dos meses;

Variáveis:

$\mathrm{E}_{\mathrm{j}} \quad=$ estoque do depósito geral, no mês j; 
$\mathrm{e}_{\mathrm{ij}} \quad=$ estoque do depósito i, no mês j;

$\mathrm{x}_{\mathrm{ij}} \quad$ =quantidade de fardos de $30 \mathrm{~kg}$ de açúcar, transportados para o depósito i no mês j;

$\mathrm{z} \quad=$ custo total de transporte;

Constantes:

$\mathrm{C}_{\mathrm{i}} \quad$ =capacidade máxima do depósito i;

$\mathrm{c}_{\mathrm{ij}} \quad=$ custo de transporte entre o depósito geral e a filial i no mês j;

$\mathrm{D}_{\mathrm{j}} \quad$ =demanda direta prevista para o mês $\mathrm{j}$;

$\mathrm{d}_{\mathrm{ij}} \quad=$ demanda no depósito i prevista para o mês j;

$\mathrm{G}=$ capacidade máxima do depósito geral;

$\mathrm{P}_{\mathrm{j}} \quad$ =produção da usina prevista para o mês j;

u =número de depósitos considerados no problema;

$\mathrm{V} \quad=$ número de meses considerados no problema.

Para facilitar a interpretação e a modelagem, não estaremos considerando estados intermediários do sistema, ou seja, estaremos supondo que os envios de açúcar acontecerão no final do mês. $\mathrm{O}$ modelo utilizado pode ser definido como:

(1) $\min z=\sum_{i=1}^{\prime \prime} \sum_{j=1}^{\prime \prime} c_{i j} x_{i j}$

Sujeito a:

(2) $e_{i(j-1)}+x_{i j} \leq C_{i}+d_{i j}(\mathrm{i}=1, \ldots, \mathrm{u} ; \mathrm{j}=1, \ldots, \mathrm{v})$;

(3) $\sum_{i=1}^{\prime \prime} x_{i j} \leq E_{j-1}+P_{j}-D_{j}(\mathrm{j}=1, \ldots, \mathrm{v})$;

(4) $e_{i(j-1)}+x_{i j} \geq d_{i j}(\mathrm{i}=1, \ldots, \mathrm{u} ; \mathrm{j}=1, \ldots, \mathrm{v})$;

(5) $E_{j-1}+P_{j}-D_{j}-\sum_{i=1}^{\prime \prime} x_{i j} \leq G(\mathrm{j}=1, \ldots, \mathrm{v})$;

(6) $e_{i(, j-1)}=e_{i 0}+\sum_{k=1}^{j-1}\left(x_{i k}-d_{i k}\right)(\mathrm{i}=1, \ldots, \mathrm{u}$; $\mathrm{j}=1, \ldots, \mathrm{v})$;

(7) $E_{j}=E_{0}+\sum_{k=1}^{j-1}\left(P_{k}-D_{k}-\sum_{i=1}^{u} x_{i k}\right)$ $(\mathrm{j}=1, \ldots, \mathrm{v})$;

(8) $x_{i j} \geq 0(\mathrm{i}=1, \ldots, \mathrm{u} ; \mathrm{j}=1, \ldots, \mathrm{v})$.

A função-objetivo (1) minimiza os custos de transporte para cada filial em cada mês considera- do. Em nosso caso u=8 e v=13; ei0 $(i=1, \ldots, u)$ e $E_{0}$ devem ser inseridas manualmente. As restrições (2) podem ser denominadas capacidade máxima de armazenagem das filiais. Indicam que para cada depósito, o estoque no final do mês (j-1) mais o que é mandado para estoque no mês atual $\mathrm{j}$, deverá ser menor ou igual a capacidade máxima de armazenagem da filial mais a demanda do mês j. As restrições (3), denominadas produção máxima da usina, especificam que para cada mês $\mathrm{j}$, a soma dos envios para as filiais deve ser menor ou igual à produção mais o estoque do depósito geral menos a demanda direta. As restrições (4) podem ser denominadas atendimento de demanda, e representam que para cada depósito, o estoque no final do mês (j-1) mais o que será enviado no mês atual $\mathrm{j}$, deverá ser maior ou igual à demanda do mês j. As restrições (5), denominadas capacidade máxima do depósito geral, especificam que o estoque no final no mês (j-1), mais a produção enviada ao depósito em j menos o envio em $\mathrm{j}$, menos $\mathrm{o}$ atendimento da demanda direta em $\mathrm{j}$ deve ser menor ou igual à capacidade máxima de armazenagem do depósito geral. As restrições (6) e (7) representam as equações de determinação de quantidade estocada de acordo com as demandas, produções e envios. E finalmente, as restrições (8) especificam que pode haver ou não envios, e que esses envios só podem ser da usina para os depósitos e não vice-versa.

$\mathrm{O}$ leitor pode verificar que as restrições (3) e (5) poderiam ser reescritas como $\mathrm{ls}_{j} \geq \sum_{i=1}^{u} x_{i j} \geq \mathrm{li}_{j}$, onde $\mathrm{ls}_{j}=E_{j-1}+P_{j}-D_{j} \mathrm{e}$ $\mathrm{li}_{j}=E_{j-1}+P_{j}-D_{j}-G$ representam respectivamente os limitantes superior e inferior da quantidade enviada. O significado é o seguinte: suponha que no final do mês $\mathrm{j}$, a usina terá disponível uma quantidade de açúcar definida pelo valor lsj; a quantidade máxima poderemos enviar é essa mesma, enquanto que a mínima que poderemos 
enviar é a quantidade disponível, menos o que o depósito geral pode absorver. Se o depósito geral for grande - G grande -, isto é, maior do que a produção de um determinado mês, pode até não haver envio de açúcar nesse mês.

Por uma característica do tipo de produto (commodity), todas as demandas existentes serão atendidas. Na realidade, empresas desse tipo definem a demanda baseando-se em sua produção. Como a demanda - do mercado - é muito maior do que a produção - da usina -, pode haver alterações de preço de modo que toda produção seja consumida pela demanda, ou seja, toda produção é escoada.

\section{Implementação Computacional}

A implementação computacional do modelo foi realizada através da utilização de um software de programação linear. Embora não tenhamos medido o tempo de solução computacional, podemos dizer que no caso analisado foi praticamente instantâneo. A flexibilidade de alteração de valores constantes também é bastante grande pois os valores podem ser inseridos através de planilhas de cálculo como o Excel ou o Lotus 12-3.

De acordo com a solicitação da empresa, pôde-se perceber na formulação que o horizonte de planejamento é de 13 meses. A idéia é atualizar o modelo mensalmente, utilizando para isso apenas os valores válidos para o primeiro mês considerado. No mês seguinte, o primeiro mês é descartado e abre-se mais um mês ao final do horizonte de planejamento.

Os dados de capacidade geralmente são facilmente obtidos, utilizando para isso a medição dos depósitos apenas. A produção da usina e as demandas previstas deverão ser estimadas. Os custos de transporte podem ser obtidos através de um estudo simples de análise de regressão para a obtenção de curvas de frete X distância. Mais recentemente com as facilidades computacionais, roteirizadores amplamente disponíveis, podem ser utilizados para a definição de custos de frete, dadas as localidades de origem e destino e o tipo de caminhão. Nesse caso deve-se tomar um cuidado especial para não desvincular a realidade da empresa do planejamento efetuado, embora por outro lado possa desvincular a empresa de uma transportadora específica.

As informações efetivamente utilizadas são as quantidades enviadas a cada um dos depósitos e o custo total de transporte em um dado mês. Os estoques são utilizados apenas para os cálculos dos envios futuros. As informações de estado atual de estoque devem ser inseridas no modelo.

As saídas computacionais podem ser de duas formas: ou na própria tela do computador, ou como um relatório impresso. A utilização da tela parece ser a opção mais interessante visto as dimensões do modelo. Para aplicações reais, o modelo pode ser considerado médio, tendo 222 variáveis e 243 valores constantes para serem avaliados ou inseridos.

\section{Avaliação Econômica do Uso do Modelo}

Por uma questão estratégica da empresa, o modelo foi implementado e avaliado mensalmente em paralelo às decisões tomadas normalmente. Em outras palavras, os transportes continuaram sendo definidos de acordo com a política convencional da empresa e o modelo foi rodado em paralelo como se estivesse sendo utilizado.

Em um período de análise de um ano, pudemos levantar uma média de economia gerada pelas decisões apontadas pelo modelo. Pelos valores encontrados, podemos verificar uma economia de aproximadamente US\$197.870,00/ 
ano. Para cada mês, coletamos dois valores relacionados aos custos de transporte: o primeiro, o valor real, obtido com o sistema convencional de tomada de decisões e o segundo, o valor hipotético, que seria o custo caso as decisões apontadas pelo modelo fossem implementadas. A Tabela 1 mostra os valores, para cada depósito, dos custos de transporte encontrados no período 6, após a implementação do modelo.

\section{Considerações Finais e Possíveis Exten- sões do Modelo}

Acreditamos que o modelo apresentado neste trabalho possa ser facilmente implementado em outras dezenas de usinas com produção e sistemas de distribuição semelhantes existentes no Brasil.

\begin{tabular}{crr}
\hline depósito & $\begin{array}{r}\text { decisão tomada com } \\
\text { o modelo }\end{array}$ & $\begin{array}{r}\text { decisão tomada sem } \\
\text { o modelo }\end{array}$ \\
\hline 1 & 19.500 & 21.000 \\
2 & 17.500 & 17.500 \\
3 & 11.100 & 11.100 \\
4 & 49.500 & 52.470 \\
5 & 96.200 & 118.400 \\
6 & 133.200 & 133.200 \\
7 & 88.800 & 111.000 \\
8 & 0 & 149.000 \\
\hline \hline total & 415.800 & 613.670 \\
\hline
\end{tabular}

Tabela 1: custos de envio de açúcar no período 6

Sem considerarmos detalhes do caso analisado, podemos dizer que o custo de modelagem e de aquisição do software foi menor do que $15 \%$ da economia anual estimada proporcionada pelo modelo. Em outras palavras, os custos de modelagem já estariam pagos pela economia gerada nos dois primeiros meses de implementação. A Figura 2 apresenta as diferenças entre os custos reais e os custos que poderiam ter ocorrido caso as decisões tomadas utilizassem o modelo apresentado anteriormente.

Considerando os custos de transporte dessa usina, podemos dizer que a redução dos custos de transporte proporcionada pela utilização do modelo, ficaria por volta de $1 \%$ do custo total de transporte.
Além dos ganhos financeiros, devemos acrescentar outros ganhos não medidos diretamente por nossa análise, como por exemplo demandas consistentemente atendidas e níveis de estoque máximo respeitados. Quando os envios excedem a capacidade do estoque, há formação de filas de caminhões aguardando o descarregamento, gerando com isso custos adicionais de multa e de estadia de caminhões ociosos. Embora isso possa parecer trivial, nem sempre é o que acontece na realidade. $\mathrm{O}$ modelo portanto pode ser utilizado como uma ferramenta de previsão de estados futuros do sistema.

Como sugestões de interesse e que poderiam ser implementadas no futuro, podemos citar a utilização de uma taxa de perda de capital para o 


\section{Diferença financeira entre as decisões tomadas com e sem o modelo}

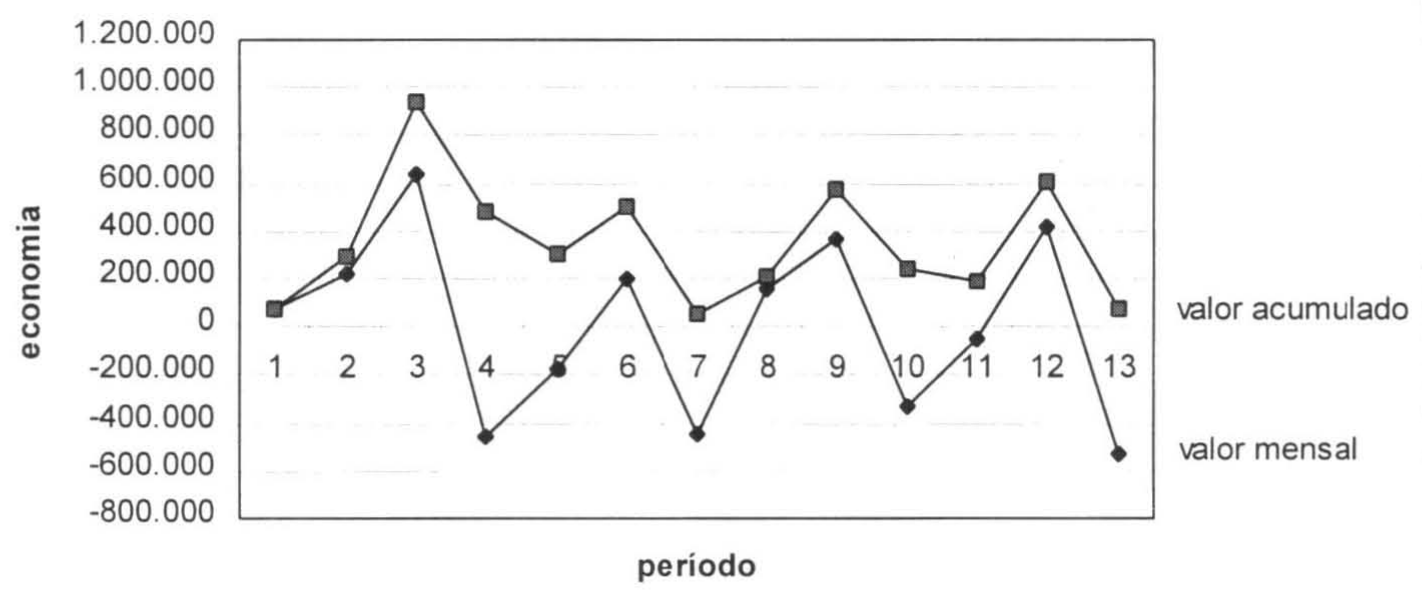

Figura 1: diferença entre os valores gerados pelo modelo e pelo sistema convencional de tomada de decisões

açúcar mantido no estoque. Os custos de estocagem, a possibilidade de se alugar depósitos para a estocagem temporária nos períodos de safra - o último dia de produção é o dia mais crítico - ou restrições com relação à embalagem ou carregamențo de produtos em caminhões também poderiam ser consideradas.

Além disso, o modelo de programação linear poderia ser estendido a um de programação quadrática onde conseguiríamos definir que quantidade produzir e a que preço vender. Outro ponto de extremo interesse para empresas desse tipo seria a análise de investimento para se acelerar a colheita no período de maior rendimento da cana, ou seja, se haveria lucro num investimento que permitisse um período de colheita menor e portanto uma maior quantidade de sacarose por unidade de massa colhida.
A consideração de alguns fatores como os citados anteriormente pode transformar o presente modelo em um outro modelo de programação não-linear ou estocástica. Entretanto, a simplicidade, a eficiência computacional e a garantia da localização do ótimo global do modelo linear são fatores importantes que devem ser considerados na elaboração de um modelo.

Agradecimentos: Os autores gostariam de agradecer aos avaliadores cujas sugestões ajudaram a melhorar a apresentação, estruturação e clareza deste trabalho. Este trabalho foi parcialmente realizado com o apoio financeiro da FAPESP - Fundação de Amparo à Pesquisa do Estado de São Paulo - por intermédio de uma bolsa de doutoramento do primeiro autor (processo número 97/11282-2). 
PRODUÇÃO

Bibliografia

1. Fisher, Marshall; Raman,

Ananth. Reducing the cost of demand uncertainty through accurate response to early sales.

Operations Research, v. 44, n. 1, pp. 87-99, 1996.

2. Taube-Netto, Miguel. Integrated planning for poultry production at Sadia. Interfaces, v. 26, n. 1, pp. 38-53, 1996.

3. Tomiya, Eduardo H. Modelo econômico de empresa sucroalcooleira. Volume 1. São Paulo, 1994. 158 pp. Dissertação (Mestrado) Escola Politécnica, Universidade de São Paulo.

4. Yoshizaki, Hugo T. Y.; Muscat, Antonio, R. N.; Biazzi, Jorge L. Decentralizing ethanol distribution in southeastern Brazil. Interfaces, v. 26, n. 6, pp. 24-34, 1996. 